\title{
SEVERE APNOEA DETECTION USING SPEAKER RECOGNITION TECHNIQUES
}

\author{
Rubén Fernández, Jose Luis Blanco, Luis A. Hernández, Eduardo López \\ GAPS: Signal, Systems \& RadioComm. Department Universidad Politécnica de Madrid, Spain \\ ruben@gaps.ssr.upm.es \\ José Alcázar \\ Respiratory Department. Unidad de Trastornos Respiratorios del Sueño Hospital Clínico Universitario Málaga, Spain. \\ jose44@separ.es
}

Doroteo T. Toledano

ATVS Biometric Recognition Group. Universidad Autonoma de Madri, Spain

doroteo.torre@uam.es

Keywords: Apnoea, Automatic Speaker Recognition techniques, GMM, nasalization

Abstract: The aim of this paper is to study new possibilities of using Automatic Speaker Recognition techniques (ASR) for detection of patients with severe obstructive sleep apnoea (OSA). Early detection of severe apnoea cases can be very useful to give priority to their early treatment optimizing the expensive and timeconsuming tests of current diagnosis methods based on full overnight sleep in a hospital. This work is part of an on-going collaborative project between medical and signal processing communities to promote new research efforts on automatic OSA diagnosis through speech processing technologies applied on a carefully designed speech database of healthy subjects and apnoea patients. So far, in this contribution we present and discuss several approaches of applying generative Gaussian Mixture Models (GMMs), generally used in ASR systems, to model specific acoustic properties of continuous speech signals in different linguistic contexts reflecting discriminative physiological characteristics found in OSA patients. Finally, experimental results on the discriminative power of speaker recognition techniques adapted to severe apnoea detection are presented. These results obtain a correct classification rate of $81.25 \%$, representing a promising result underlining the interest of this research framework and opening further perspectives for improvement using more specific speech recognition technologies

\section{INTRODUCTION}

Obstructive sleep apnoea (OSA) is a highly prevalent disease (Puertas et al., 2005), affecting an estimated $2-4 \%$ of population between the ages of 30 and 60 years. It is characterized by recurring episodes of upper airway obstruction during sleep, and it is usually associated with loud snoring and increased daytime sleepiness. OSA is a serious threat to an individual's health if not treated (Puertas et al., 2005). OSA is a risk factor for cardiovascular disease (Coccagna et al., 2006), it is usually related to traffic accidents caused by somnolent drivers (Puertas et al., 2005; Lloberes et al., 2000), and it can lead to a poor quality of life and impaired work performance. A full overnight sleep study is usually required to diagnose OSA; referred to as polysomnography it involves the recording of neuroelectrophisiological and cardiorespiratory variables. Polysomnography is an expensive and time-consuming test so patients usually have to suffer a waiting list of several years before the test is done (Puertas et al., 2005). At present, the most effective and widespread treatment for OSA is nasal CPAP (Continuous Positive Airway Pressure) which prevents apnoea episodes providing a pneumatic splint to the airway.

In this article we investigate the acoustical characteristics of patients' speech with OSA with the purpose of relying on an automatic system for severe 
apnoea voice assessment. The acoustic properties of speakers suffering from obstructive sleep apnoea are not clear, and not much research has been made on it. However, there are some studies pointing at possible abnormalities in phonation, articulation and resonance (Fox \& Monoson, 1989). In a previous research, we designed and collected an apnoea speech database (Fernández et al., 2008) based on both, the review of previous research in this field, and a preliminary manual contrastive study performed on a small group of healthy subjects and apnoea patients. This contrastive study helped us to identify phonetically related acoustic characteristics of patients' speech with OSA.

In this work, we explore the possibilities for the adaptation of Automatic Speaker Recognition techniques (ASR) to severe apnoea cases diagnosis. To our knowledge this represents a pioneer research on automatic severe OSA diagnosis using signal processing algorithms on continuous speech. The proposed method intends to be complementary to existing OSA diagnosis methods (i.e. Polysomnography) and clinician perceptual judgments, to help in an early detection of these cases. Early severe OSA detection can enable more efficient medical protocols, giving higher priority to more risky cases (there is a high risk of car accident due to somnolence in severe apnoea patients) and thus optimizing both social benefits and medical resources. The proposed system is based on Gaussian Mixture Models (GMMs), which represents the state-of-the-art for speaker recognition (Reynolds et al., 2000).

More specifically, our work is mainly focused on the nasality factor, since it has been traditionally identified as an important feature in the acoustic characteristics of apnoea speakers. The term nasalization can refer to two different phenomena in the context of speech; hyponasality and hypernasality. The first type occurs if no nasalization is produced when the speech sound should actually be nasal. This is the case when for example having a cold. Hypernasality is nasalization during the production of non-nasal (voiced oral) sounds. The interested reader can find an excellent reference work in (Pruthi, 2007). Up to now no signal processing and pattern recognition techniques have been applied to the analysis hyponasal or hypernasal continuous speech in OSA patients, therefore this will represent one of the aims of our research.

The rest of this paper is organized as follows: Section 2 addresses the physiological and acoustic characteristics in OSA patients. The speech database used in our experimental work is described in Section 3. Section 4 explores the possibilities of using GMM approaches to study and model nasalization in OSA patient's speech. Application of speaker recognition technologies for automatic severe apnoea detection is presented in Section 5. Finally, some conclusions and future research are given in Section 6.

\section{PHYSIOLOGICAL \& ACOUSTIC CHARACTERISTICS IN OSA SPEAKERS}

Nowadays is still not clear neither the articulatory/physiologically settings nor the acoustic characteristics of speech in apnoea speakers. Most of the more valuable information in this field can be found in Fox and Monoson work (Fox \& Monoson,, 1989) where a perceptual study with skilled judges was presented comparing voices from apnoea patients and a control group (referred to healthy subjects). This study showed that although differences between both groups of speakers were found, acoustic cues for these differences are somewhat contradictory and unclear. What seemed to be clear was that apnoea group had abnormal resonances that might be due to altered structure or function of the upper airway, and theoretically, this anomaly should result not only in a respiratory but also in a speech dysfunction. Consequently, the presence of speech disorder in a OSA population should be expected, and it could include abnormalities in articulation, phonation and resonance:

- Articulatory anomalies: Fox and Monoson stated that neuromotor dysfunction could be found in a sleep apnoea population as a "lack of regulated innervations to the breathing musculature or upper airway muscle hypotonus" This dysfunction is normally related to speech disorders, especially dysarthria. There are several types of dysarthria, resulting in various different acoustic features. All types of dysarthria affect the articulation of consonants and vowels causing the slurring of speech. Another common feature in apnoea patients is hypernasality and problems with respiration.

- Phonation anomalies: The phonation anomalies may be due to the fact that the heavy snoring of 
sleep apnoea patients can cause the inflammation in the upper respiratory system and affect the vocal cords.

- Resonance anomalies: The analysis of resonance characteristics for the sleep apnoea group in (Fox \& Monoson, 1989) did not yield any clear conclusion. These authors only conclude that resonance abnormalities in apnoea patients could be perceived as hyponasality or hypernasality. It is only recently that resonance disorder affecting speech sound quality has been associated with vocal tract damping features distinct from airflow in balance between the oral and nasal cavities. The term applied to this speech disorder is "cul-de-sac" resonance and is used for a type of hyponasality.

\subsection{Initial contrastive acoustic study}

A manual contrastive study of an initial version of our apnoea speech database was made. A similar revision can be found in (Fiz et al, 1993) where an acoustic spectral analysis was applied to sustained vowels to detect possible OSA cases. Nevertheless, this study doesn't investigate all the possible acoustic characteristics in apnoea patients' voice because the use of sustained vowels excludes the study of other acoustic clues present in continuous speech and measures based on the comparison between different linguistic contexts (Parsa \& Jamieson, 2001).

For our preliminary study, a group of 16 speakers, eight in the apnoea group and eight in the control group, were recorded. All the speakers uttered a same group of 25 phonetically balanced sentences; they were extracted from the phonetic corpus of Albayzin database (Moreno et al., 1993), a resource for speech recognition technology in Spanish. The contrastive study was performed by visual comparison of frequency representations (mainly spectrographic, pitch, energy and formant analysis) of apnoea and control group speakers.

Following some conclusions of previous research on acoustic characteristics of apnoea speakers we checked nasality feature. The existence and the size of one extra low frequency formant was studied (Glass \& Zue, 1985) as indicator of nasalization, but no perceptual differences between the groups could be found. As discussed in (Fox \& Monoson, 1989), this fact could be explained by the perceptual difficulty in classifying the apnoea speakers' voice as hyponasal or hypernasal disorder. However, studies on specific linguistic contexts were also made and differences in the variation in nasalization degree between different linguistic contexts (nasal and non-nasal context) of both groups were found, there being a tendency for the apnoea speakers to have smaller differences between those contexts. This is indeed very interesting. A hypothesis has been that apnoea speakers might have an overall higher nasality level due to velopharyngeal dysfunction, and that the differences between oral would be small, because the oral vowels would also be nasalized. One explanation could be that the control of the velopharyngeal mechanism is lower for the apnoea speakers, which could lead to difficulties in making changes of nasality, although the absolute nasalization might be either high or low

Another interesting conclusion of our study was, related to resonance anomalies, when we compare the distance between the second and third formant for the /i/ vowel, clear differences between the apnoea and control groups were found. Apnoea speakers showed a higher distance and this was especially clear in diphthongs where /i/ was stressed, for example in the Spanish word "Suiza" ('suj $\theta a$ ). According to (Hidalgo \& Quillis, 2002) the position of the third formant might be related to the degree of velopharyngeal opening. Lowering of velum gives rise to higher frequencies of the third formant.

\section{APNOEA DATABASE}

The database collection was performed in the Respiratory Department at Hospital Clínico Universitario of Málaga, Spain. The database was composed of about 80 male subjects; half of them with severe sleep apnoea (AHI > 30), and the other half healthy subjects or with mild OSA (AHI $<10)$. Subjects in both groups have similar physical characteristics such as age and body mass index. Furthermore, speech material for the apnoea group was recorded and collected in two different sessions: one just before being diagnosed and the other after several months under CPAP treatment. The analysis of speech characteristics in these two sessions will allow studying the evolution of apnoea patient's voice characteristics after the treatment.

\subsection{Speech and Image Collection}

Speech signal was recorded with a sampling frequency of $48 \mathrm{kHz}$ in an acoustically isolated booth. Recording equipment was a standard laptop computer equipped with a SP500 Plantronics 
headset microphone that includes $\mathrm{A} / \mathrm{D}$ conversion and digital data exchange through USB-port.

Additionally, for each subject in the database, two facial images (frontal and lateral views) were collected under controlled illumination conditions and over a white flat background. A conventional digital camera was used to obtain images in a 24-bit RGB format, without compression and with a $2272 \times 1704$ resolution. We decide to collect visual information because OSA is associated to a variable combination of different anatomic factors and a visual exam of the patient is suggested as first step in the examination of a subject with clinical suspicion of OSA (Puertas et al., 2005), valuing the morphotype (i.e. obesity, short neck, etc.) and facial features (i.e. malocclusions referred to bad bites, etc.). To our knowledge, no research has been made on finding evidences that could relate facial features extracted through image processing of patients' face pictures to severe apnoea cases diagnosis. Thus we decided to include both frontal and lateral pictures of each patient's face making it possible to research on this challenging topic.

\subsection{Speech Corpus}

The speech corpus consists in four sentences repeated three times and a sustained vowel /a/ also repeated three times. The four sentences were designed trying to cover all the relevant linguistic contexts where physiological OSA features could have higher impact on specific acoustic characteristics of particular phonemes. Particular emphasis has been put on:

- Sentence design that allows intra-speaker variation measurements; that is to measure differential voice features for each speaker, as, for example, the nasalization degree between vowels inside and outside nasal contexts.

- Continuous voiced sounds to measure irregular phonation patterns related to muscular fatigue of apnoea patients.

- Voiced sound in the context of guttural phonemes to analyse the specific impact of possible articulatory dysfunctions in the pharyngeal region.

- A broad range of vowel sounds to allow the accurate modelling of the articulation space and other measures related to our preliminary contrastive study.
All sentences where designed to exhibit a similar melodic structure, and speakers where requested to read them following this rhythmic structure. We hope this controlled rhythmic recording procedure could help in avoiding non-relevant inter-speaker linguistic variability. Each sentence is presented in the following paragraphs where the melodic groups have been underlined.

1. Francia, Suiza y Hungría ya hicieron causa común. ('fraN $\theta j a$ 'suj $\theta$ a i uy 'gri a ya j'Oje roy 'kaw sa ko 'mun).

2. Julián no vio la manga roja que ellos buscan, en ningún almacén. $(x u$ 'ljan no

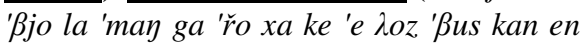
niy 'gun al ma 'ken).

3. Juan no puso la taza rota que tanto le gusta en el aljibe. ( $x$ wan no 'pu so la 'ta $\theta a$ 'ro ta ke 'taN to le ' $\gamma$ us ta en el al 'xi ße).

4. Miguel y Manu llamarán entre ocho y nueve y media. ( $m i$ 'yel $i$ 'ma nu $\lambda a$ ma 'ran 'eN tre 'o t fo i 'nwe ße i 'me ðja).

First phrase includes stressed vowels /i/ in diphthongs where (according to our preliminary contrastive study, see 2.1) differences between the second and third formant could be distinctive in apnoea speakers. Second and third phrases, both negatives, have similar grammatical and intonation structure. They could be useful to make contrastive studies of vowels in different linguistic contexts. Some examples of these contrastive pairs are: a nasal context, "manga roja" ('may ga 'ro $x a$ ) versus a neutral context, "taza rota" ('ta $\theta a$ 'ro ta). As we mention in previous sections, these contrastive analyses could be indeed very interesting to confirm that apnoea speakers might have an overall higher nasality level and smaller intra-speaker differences between non-nasal and nasal vowels due to velopharyngeal dysfunction. Fourth phrase includes a relatively long sentence mainly composed of voiced sounds read as one single melodic group in order to discover possible phonation abnormalities during the sustained generation of voiced sounds. The three repetitions of the sustained vowel /a/, including onset and offset, could be used to study phonation measures (i.e. jitter and shimmer) that detect the variations in amplitude and frequency of the speakers, but in a more controlled way. 


\section{GMM FOR OSA VOICE MODELLING}

In this section we present an initial experimentation directed to evaluate the capabilities of using Gaussian Mixture Models techniques (GMM) to model perceptual acoustic characteristics described in previous research (Fox \& Monoson, 1989) and corroborated in our initial contrastive study.

In particular, as our speech database was designed to allow a detailed contrastive analysis of vowels in oral and nasal phonetic contexts, we focussed on reporting perceptual differences related to resonance anomalies and perceived as hyponasality or hypernasality. To that purpose, GMM-based approach techniques were applied to confirm these differences in nasalization degree between different linguistic contexts, as was already described in Section 2 and was revealed in the initial contrastive study.

\subsection{GMM Training and Testing protocol}

Gaussian Mixture Models (GMMs) and adaptation algorithms are effective and efficient pattern recognition techniques suitable for sparse speech data modelling in Automatic Speaker Recognition systems (Reynolds et al., 2000) that we will apply for apnoea voice modelling. In our experimental framework, the ASR open source tool BECARS (Blouet et al., 2004) was used. Details on parameterization, model training and classification phases for the BECARS baseline system are the following:

- Parameterization consists in extracting information from speech signal (in our case, 39 MEL frequency cepstral coefficients -MFCC-, delta MFCCs and delta-delta MFCCs).

- GMMs for apnoea and control groups were trained as follows. First, a universal background model (UBM) was trained on a subcorpus of Albayzin Database (Moreno et al., 1993), thus entirely separated from the apnoea speech database. Next, speech from apnoea and control group speakers was used to train the apnoea and control group GMM models. Both models were trained using MAP adaptation from the UBM. Only means were adapted (as classically done in speaker verification). This technique increases robustness of the models, especially when little speech material is available. Obviously, speech data from speakers for the model training was not included in the test set.

For testing purposes, in order to increase the number of tests and thus to improve the statistical relevance of our results, the standard leave- $x$-out testing protocol was used. This protocol consists in discarding $\mathrm{x}$ speakers from the experimental set, training some models on the remaining data and testing with that $\mathrm{x}$ speakers. This scheme is replicated until reaching a sufficient number of tests.

\subsection{OSA resonance anomalies study using GMMs}

With the aim of testing the capabilities of using GMM technique to perform contrastive analysis on perceived resonance anomalies, two GMMs for each apnoea and healthy speaker were modelled using speech from nasalized and non-nasalized vowels. Both, nasal and non-nasal GMM models were trained using a similar approach described in section 4.1; MAP adaptation from a generic vowel UBM model trained using Albayzin. These two nasal/nonnasal GMM models were used to quantify the acoustic differences between nasal and non-nasal contexts for both apnoea and control groups. The smaller difference between nasal/non-nasal GMM models, the more similar are both nasalized and nonnasalized vowels; thus revealing the presence of resonance anomalies. As distance measure between nasal/non-nasal GMM models, a fast approximation of the Kullback-Leibler (KL) divergence for Gaussian Mixture Models was used (Do, 2003). This distance is used in ASR to define cohorts or groups of speakers producing similar sounds.

As can be seen in Figure 1, significant differences in the nasal/non-nasal GMM distances were found for control and apnoea groups. This result clearly shows that acoustic differences between oral and nasal vowels are smaller in apnoea speakers and confirms the trend to an overall higher nasality level revealed in previous research. As a result, GMM approach appears as an appropriate and suitable speech technique for further research on modelling and detecting severe apnoea speakers. 


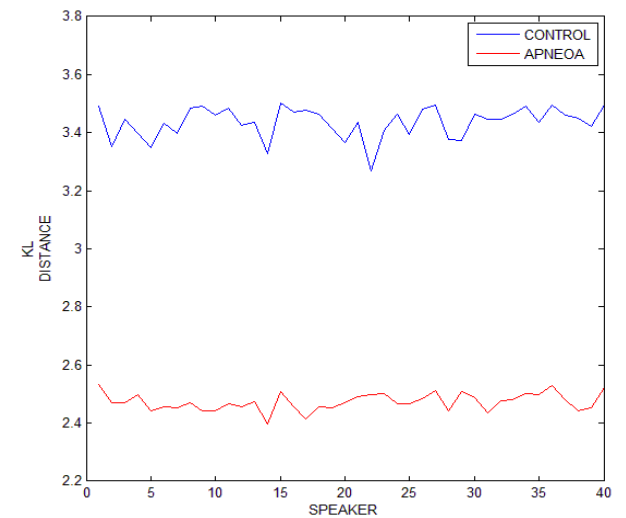

Figure 1: Kullback distances differences between nasal and non-nasal models for apnoea and control speakers.

\section{GMM-BASED SPEAKER RECOGNITION TECHNIQUES FOR SEVERE APNOEA DETECTION}

As we have seen in the previous section, GMM approach is able to model some of the already described resonance abnormalities perceived in apnoea speakers. Now, this experimental set-up was intended to test the potential of applying standard ASR techniques to the automatic diagnosis of apnoea disease. A speaker verification system is a supervised classification system able to discriminate speech signals into two classes (genuine and impostor). In our case, the classes will not correspond to a given speaker, but a sleep apnoea class and a control class (referred to healthy subjects). This method is suitable for keeping track the evolution of voice dysfunction among the patients, is easy-to-use, quick, non-invasive for the patients and affordable for the clinicians. The proposed method is not expected to replace the existing OSA diagnosis methods, but to help in an early detection of severe apnoea cases.

Following a similar approach already used for other pathological voice assessment studies (Fredouille et al., 2005), GMM models representing the apnoea and control classes were built as follows:

- The pathological and control GMM models were trained from the generic UBM model thanks to the MAP adaptation technique using standard leave- $x$-out technique, in a similar method as described above (Section 4).

- During the apnoea/non-apnoea detection phase, an input speech signal corresponding to the utterance of a speaker to be diagnosed is presented to the system. The parameterised speech is then processed with each apnoea and control GMM model generating two likelihood scores. From these two scores an apnoea/control decision is made.

In these initial experiments the task of control and apnoea voice classification has been investigated. Table 1 provides the correct classification rates obtained. An overall correct classification of 81.25 $\%$ is reached on this task.

\begin{tabular}{|c|c|c|c|}
\hline $\begin{array}{c}\text { Correct } \\
\text { Classi. }\end{array}$ & $\begin{array}{c}\text { Control } \\
\text { Group }\end{array}$ & $\begin{array}{c}\text { Apnoea } \\
\text { Group }\end{array}$ & Overall \\
\cline { 2 - 4 } Rate in \% & $\begin{array}{c}\mathbf{7 7 , 5 0 \%} \\
\mathbf{( 3 1 / 4 0 )}\end{array}$ & $\begin{array}{c}\mathbf{8 5} \% \\
\mathbf{( 3 4 / 4 0 )}\end{array}$ & $\begin{array}{c}\mathbf{8 1 , 2 5} \% \\
\mathbf{( 6 5 / 8 0 )}\end{array}$ \\
\hline
\end{tabular}

Table 1: Correct Classification Rate.

Table 2 shows the statistical measures of the performance for the same task (Fisher's exact test $\mathrm{p}<0.0001$ revealed statistically significant). Results are presented in terms of Sensitivity: proportion of positives which are correctly identified as such; Specificity: proportion of negatives which are correctly identified; Positive Predictive Value: proportion of patients with positive test results who are correctly diagnosed; Negative Predictive Value: proportion of patients with negative test results who are correctly diagnosed.

\begin{tabular}{|c|c|c|c|}
\hline Sensitivity & Specificity & $\begin{array}{c}\text { Positive } \\
\text { Predictive } \\
\text { Value }\end{array}$ & $\begin{array}{c}\text { Negative } \\
\text { Predictive } \\
\text { Value }\end{array}$ \\
\hline $\mathbf{7 7 , 5 0 \%}$ & $\mathbf{8 5} \%$ & $\mathbf{8 3 , 7 8} \%$ & $\mathbf{7 9 , 0 6} \%$ \\
$(\mathbf{3 1 / 4 0 )}$ & $\mathbf{( 3 4 / 4 0 )}$ & $\mathbf{( 3 1 / 3 7 )}$ & $\mathbf{( 3 4 / 4 3 )}$ \\
\hline
\end{tabular}

Table 2: Sensitivity, Specificity, Positive Predictive Value and Negative Predictive Value.

Considering the correct classification rates achieved, there are some aspects to comment. The results are encouraging and it seems that apnoea information may be caught by a GMM based- 
approach, even with few training speech. These promising results have been obtained without particular attention to the choice of acoustic parameters used, so best results can be expected with a representation of the audio data optimized for pathology discrimination. Evidently, these experiments have to be validated with a higher population, but the results have already given us a preliminary notion of the discriminative power of this approach for the automatic diagnosis of severe apnoea cases.

\section{CONCLUSIONS AND FUTURE RESEARCH}

In this paper the acoustic properties of speakers with severe obstructive sleep apnoea (OSA) and the adaptation of standard ASR techniques for detection and modelling of these patients have been presented. This study represents a pioneer research in the field of automatic diagnosis of OSA disease.. Preliminary experimental results on the speech database collected using state-of-the-art GMM speaker recognition techniques shows that it is worth continuing the research on this area. Related to nasality factor as an important feature in the acoustic characteristics of apnoea speakers, GMM approach have confirmed that there are significant differences between apnoea and control group on the relative nasalization degree between different linguistic contexts. Therefore, future research will be focused on exploiting this information in order to use it for the automatic apnoea diagnosis. Furthermore, best results can be expected with a representation of the audio data optimized for pathology discrimination

On the other hand, and bearing in mind the speech database design criteria, we propose the use of other acoustic measures usually applied over pathological voices (jitter, HNR, etc.). These techniques could also be applied over different linguistic and phonetic contexts, and could be fussed to GMM approach to improve our initial discrimination results.

\section{ACKNOWLEDGEMENTS}

The activities described in this paper were funded by the Spanish Ministry of Science and Technology as part of the TEC2006-13170-C02-01 project. The authors would like to thank the volunteers at Hospital Clínico Universitario of Málaga, Spain, and to Guillermo Portillo who made the speech and image data collection possible.

\section{REFERENCES}

Blouet, R., Mokbel, C., Mokbel, H., Sanchez Soto, E., Chollet, G., \& Greige, H. (2004). BECARS: a Free Software for Speaker Verification. In Proceedings of The Speaker and Language Recognition Workshop, ODYSSEY, pp 145-148.

Coccagna, G., Pollini, A., \& Provini, F. (2006). Cardiovascular disorders and obstructive sleep apnea syndrome. In Clinical and Experimental Hypertension Vol. 28:217-24.

Do, M. N., (2003) Fast approximation of KullbackLeibler distance for dependence trees and Hidden Markov Models. IEEE Signal Processing Letter 10, 115-118.

Fernandez R., Hernández L. A., López E., Alcázar J., Portillo G., \& Toledano D. T. (2008). Design of a Multimodal Database for Research on Automatic Detection of Severe Apnoea Cases. In Proceedings of 6th Language Resources and Evaluation Conference. LREC, Marrakech.

Fiz, J.A., Morera, J., Abad, J., Belsulnces, A., Haro, M., Fiz, J.I., Jane, R., Caminal, P., \& Rodenstein, D. (1993). Acoustic analysis of vowel emission in obstructive sleep apnea. In Chest Journal; 104: 1093 - 1096.

Fox, A.W., \& Monoson, P.K. (1993). Speech dysfunction of obstructive sleep apnea. A discriminant analysis of its descriptors. In Chest Journal; 96(3): 589-595.

Fredouille, C., Pouchoulin, G., Bonastre, J.F., Azzarello, M., Giovanni, A., \& Guio, A. (2005). Application of Automatic Speaker Recognition techniques to pathological voice assessment (dysphonia). In Proceeding of 9th European Conference on Speech Communication and Technology, Interspeech 2005, Lisboa, p. 149-152.

Glass, J., \& Zue, V. (1985). Detection of nasalized vowels in American English. In Proceedings of Acoustics, Speech, and Signal Processing, IEEE International Conference on ICASSP, Volume: 10, p. 1569- 1572.

Hidalgo, A., \& Quilis, M. (2002). Fonética y fonología españolas. Editorial Tirant blanch.

Lloberes, P., Levy, G., Descals, C., et al. (2000). Self-reported sleepiness while driving as a risk 
factor for traffic accidents in patients with obstructive sleep apnoea syndrome and in nonapnoeic snorers. In Respiratory Medicine 94: 971-6.

Moreno, A., Poch, D., Bonafonte, A., Lleida, E., Llisterri, J., Mariño, J.B., \& Naude, C. (1993). ALBAYZIN Speech Database: Design of the Phonetic Corpus. In Proceedings of Eurospecch 93. Berlin, Germany, 21-23. Vol. 1 pp. 175-178.

Parsa, V., \& Jamieson, D. G. (2001). Acoustic discrimination of pathological voice : Sustained vowels versus continuous speech. In Journal of Speech, Language, and Hearing Research ISSN 1092-4388, vol. 44, no2, pp. 327-339 (1 p.1/4).

Pruthi T. (2007) Analysis, vocal-tract modeling and automatic detection of vowel nasalization. Doctor Thesis at the University of Maryland.

Puertas, F.J., Pin, G., María, J.M., \& Durán, J. (2005). Documento de consenso Nacional sobre el síndrome de Apneas-hipopneas del sueño (SAHS). Grupo Español De Sueño (GES).

Reynolds, D.A., Quatieri, T.F., \& Dunn, R.B. (2000). Speaker verification using adapted gaussian mixture models. In Digital Signal Processing 10: $19-41$. 